专家推介: 本文研究了 MCP 输入增强技术 (Mi2 技术, MCP input intensified) 对 MCP 性能的影响。通过在 $\mathrm{MCP}$ 输入端上镀制一层高二次电子发射系数的介质膜层，覆盖 MCP 输入端原有的金属电极，提高了 MCP 输 入信号的利用率，因此提高了像增强器增益、分辨力以及信噪比。

\title{
MCP 输入增强膜对像增强器主要性能的影响研究
}

\author{
曾进能 ${ }^{1,2}$, 李廷涛 ${ }^{1}$, 常 乐 $^{1}$, 龚燕妮 ${ }^{1}$, 赵伟林 ${ }^{1}$, 赵 恒 $^{1}$, \\ 张 俊 $^{1}$, 褚祝军 ${ }^{1}$, 李顺平 ${ }^{1}$, 李晓峰 ${ }^{1}$
}

（1. 北方夜视技术股份有限公司，云南 昆明 650217；2. 微光夜视技术重点实验室，陕西 西安 710065）

摘要: MCP 是一种超快响应的电子倍增器, 在像增强器和光电倍增管中有广泛应用。本文首先介绍 了 MCP 输入增强膜原理, 之后利用真空镀膜方法在 MCP 的输入端镀制了一层具有高二次电子发射系 数的膜层, 并通过面电阻、XPS 表征了膜层特性。通过试验, 对比测量了镀膜 MCP 和常规 MCP 像 增强器的信噪比、 MCP 增益以及像增强器分辨力, 测量结果表明, 镀膜 MCP 像增强器的信噪比、 MCP 增益较常规 MCP 像增强器的信噪比、MCP 增益均有提高, 但像增强器分辨力有所下降。常规 MCP 像增强器的信噪比平均为 25.27、MCP 增益平均为 209.5 、像增强器分辨力平均为 $61 \mathrm{lp} / \mathrm{mm}$, 而 镀膜 MCP 像增强器的信噪比平均为 29.53、 $\mathrm{MCP}$ 增益平均为 450.5、像增强器分辨力平均为 54.75 $1 \mathrm{p} / \mathrm{mm}$ 。镀膜 MCP 像增强器信噪比和 MCP 增益提高的原因是 MCP 输入端镀膜以后, 表面二次电子 发射系数提高。另外由于 MCP 输入端表面二次电子发射系数提高, 导致镀膜 MCP 输入端表面散射电 子数量的增加, 使得镀膜 MCP 像增强器分辨力有所下降。

关键词: 像增强器; 微通道板; 分辨力; 信噪比; $\mathrm{MCP}$ 增益; 二次电子

中图分类号：O462.3 文献标识码：A 文章编号：1001-8891(2020)08-0735-07

\section{Effect of MCP Input Enhancement Film on Image Intensifier Performance}

\author{
ZENG Jinneng $^{1,2}$, LI Tingtao ${ }^{1}$, CHANG Le ${ }^{1}$, GONG Yanni ${ }^{1}$, ZHAO Weilin ${ }^{1}$, ZHAO Heng ${ }^{1}$, \\ ZHANG Jun ${ }^{1}$, CHU Zhujun ${ }^{1}$, LI Shunping ${ }^{1}$, LI Xiaofeng ${ }^{1}$ \\ (1. North Night Vision Technology Co. Ltd, Kunming 650217, China; \\ 2. Science and Technology on Low-light-level Night Vision Laboratory, Xi'an 710065, China)
}

\begin{abstract}
A microchannel plate(MCP)is a superfast response electron multiplier that is widely used in image intensifiers and photomultipliers. Herein, first, the principle of MCP input enhancement film is introduced; subsequently, a film with a high secondary electron emission coefficient is deposited on the input end of the MCP via vacuum coating, and the characteristics of the film are characterized by surface resistance and X-ray photoelectron spectroscopy(XPS). Through experiments, the signal-to-noise ratio, MCP gain, and image intensifier resolution of the coated MCP and conventional MCP image intensifier are measured. The results show that the signal-to-noise ratio of the image intensifier and the MCP gain of the coated MCP are higher than those of the conventional MCP; however, the resolution of the image intensifier is lower. For the conventional and coated MCP image intensifiers, the signal-to-noise ratios are 25.27 and 29.53, respectively; the average MCP gains are 209.5 and 450.5, respectively; the average resolutions of the image intensifier are 61 and $54.75 \mathrm{lp} / \mathrm{mm}$, respectively. The increase in the signal-to-noise ratio of the image intensifier and MCP
\end{abstract}


gain are due to the increase in the surface secondary electron emission coefficient after the MCP input is coated. In addition, owing to the increase in the secondary electron emission coefficient of the MCP input surface, the number of scattered electrons on the MCP input surface increases, thereby decreasing the resolution of the MCP image intensifier.

Key words: image intensifier, MCP, resolution, signal to noise ratio, MCP electronic gain, secondary electron

\section{0 引言}

像增强器是微光夜视仪的核心器件, 广泛用于 夜间成像观察、弱光探测等装备。像增强器的工作 原理 ${ }^{[1-3]}$ 是将目标的光学图像通过光电阴极 ${ }^{[4-6]}$ 转变 为光电子图案, 光电子图案经过 $\mathrm{MCP}$ 倍增后, 通过 苂光屏显示为光学图像。 $\mathrm{MCP}^{[7-8]}$ 具有电子倍增数量 高、体积小、重量轻、使用电压低等优点, 被广泛应 用到像增强器和电子探测器中。MCP 是由数以万计的 微通道组成的二维阵列, 微通道阵列是利用硅酸铅玻 璃拉制技术加工形成, 经过氢还原处理, 使玻璃基体 中的一部分 $\mathrm{PbO}$ 还原成游离态的 $\mathrm{Pb}$, 还原结束后, 微通道内壁表面的 $\mathrm{PbO} 、 \mathrm{~K}_{2} \mathrm{O} 、 \mathrm{Na}_{2} \mathrm{O}$ 为二次电子发射 层 $^{[9]}$, 其二次电子发射系数 $\delta$ 在 $2 \sim 3$ 之间。

衡量 MCP 性能的主要技术指标是对电子的探测 效率和倍增能力, $\mathrm{MCP}$ 的开口面积比是决定该指标的 关键因素, 目前国内常用的 MCP 开口面积比为 $65 \%$ 。 普通像增强器工作时, 光电阴极发出的光电子有很大 一部分被 MCP 输入端 Ni-Cr 导电层吸收, 成为无效 光电子, 因此降低了 $\mathrm{MCP}$ 对电子的探测效率。为提 高 MCP 对电子的探测效率, 研究人员采取了一系列 措施, 常用的措施是在 MCP 输入端导电层表面镀制 一层具有高二次电子发射系数、性能稳定可靠的功能 薄膜, 俗称 “输入增强膜”。电子轰击输入增强膜产 生一定数量的二次电子, 在均匀电场的作用下部分二 次电子能够进入 MCP 的通道, 此时输入增强膜以间 接的方式提高了 $\mathrm{MCP}$ 对电子的探测效率 ${ }^{[10]}$ 。

本文通过实验验证和理论分析相结合的方法, 在 $\mathrm{MCP}$ 输入端表面制作一层材料为 $\mathrm{MgO}$ 的输入增强薄 膜, 封装为像增强器后, 测量和分析像增强器的分辨 力、 $\mathrm{MCP}$ 增益以及信噪比的变化, 为提高像增强器性 能奠定了良好的基础。

\section{1 输入增强膜工作原理}

普通的 MCP 工作时, 光电阴极发射出的光电子 到达 MCP 输入面时, 其去向主要有 3 种情况: (1)直 接进入通道成为有效光电子; (2)进入非开口区导电层
表面和通道前端导电层表面被吸收, 成为无效光电 子; (3)在非开口区导电层表面和通道前端导电层表面 发生弹性碰撞, 形成散射光电子。

$\mathrm{MCP}$ 镀制输入增强膜后, 光电阴极发射出的光电 子到达 $\mathrm{MCP}$ 输入面时, 会发生以下主要运动 (如图 1 所示): (1)直接进入通道成为有效光电子; (2)进入非 开口区和通道前端的输入增强膜, 激发其产生二次电 子。大部分二次电子在均匀电场作用下能够进入通道, 成为有效电子, 提高了 $\mathrm{MCP}$ 对电子的探测效率。

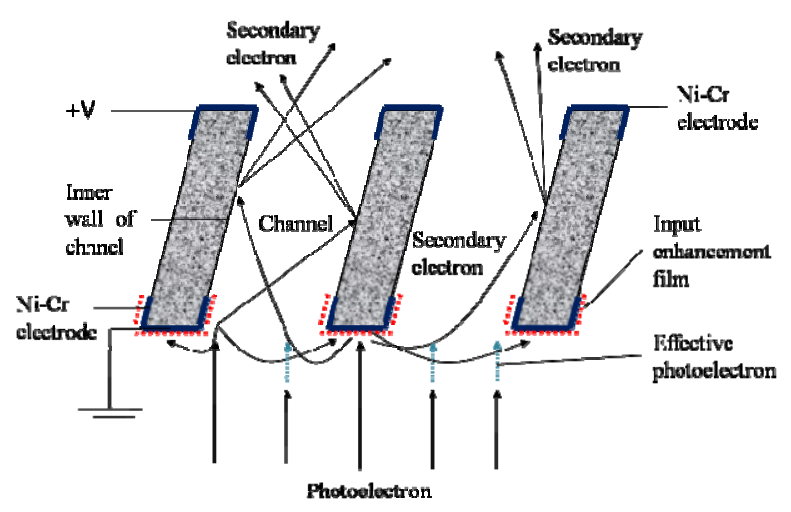

图 1 输入增强膜工作原理图

Fig.1 Working principle of input enhancement film

输入增强膜的二次电子发射系数越高, MCP 的探 测效率也就越高, 因此输入增强膜需要具有高二次电 子发射系数。目前已知具有高二次电子发射系数的材 料有很多, 如氧化硅 $\left(\mathrm{SiO}_{2}\right)$ 、氧化镁 $(\mathrm{MgO})$ 、氧化 铝 $\left(\mathrm{Al}_{2} \mathrm{O}_{3}\right)$, 甚至是氧化钙 $(\mathrm{GaO})$ 、氧化锌 $(\mathrm{ZnO})$ 、 氟化钙 $\left(\mathrm{CaF}_{2}\right)$ 、氯化钠 $(\mathrm{NaCl})$ 等, 以上 7 种材料 的二次电子发射系数分别是 $3.90 、 4.50 、 3.10 、 5.10$ 、 $6.65 、 4.82 、 6.50^{[11]}$ 。像增强器内部为高真空状态, 输 入增强膜还需要具有在高温下放气量小、稳定性高的 基本要求。经过分析, $\mathrm{MgO}^{[12]}$ 薄膜具有放气量小、二 次电子发射系数高等优点, 所以选择 $\mathrm{MgO}$ 作为输入 增强膜的材料。

\section{2 实验与结果分析}

\section{1 实验方案}


实验用 $\mathrm{MCP}$ 来自于同一屏段, 通道直径为 $7 \mu \mathrm{m}$ 、 开口面积比为 $65 \%$ 、斜切角为 $7^{\circ}$ 、输入端导电层深度 约为 $0.5 d$ ( $d$ 为通道直径)。取 12 片 $\mathrm{MCP}$ 平分为 $\mathrm{A}$ 、 $\mathrm{B}$ 两组, 其中 $\mathrm{A}$ 组 MCP 采用真空镀膜法镀制一定厚 度的输入增强膜, 材料为 $\mathrm{MgO}$, 深度为 $0.5 d$, 即刚好 覆盖输入端导电层, B 组 MCP 不做镀膜处理。从 A 组 MCP 和 B 组 MCP 中各取 4 片在相同环境下制作 相同型号的像增强器 (装配 A 组 MCP 的像增强器称 为 $\mathrm{A}$ 组像增强器, 装配 $\mathrm{B}$ 组 $\mathrm{MCP}$ 的像增强器称为 $\mathrm{B}$ 组像增强器), 并且在相同条件下测试其分辨力、 $\mathrm{MCP}$ 增益、信噪比, 比较 MCP 镀膜后像增强器性能的变 化。每组剩余的 2 片 MCP 用于测试分析。

\section{$2.2 \mathrm{MgO}$ 薄膜分析}

1) 面电阻

MCP 正常工作时, 对输入面电阻值有具体要求, 因此 MCP 镀制输入增强膜后需要进行面电阻的测试。 在常温常压下, 测量 $\mathrm{A}$ 组和 B 组中未使用的 4 片 MCP 输入端的面电阻。测量仪器是美国福禄克公司生产的 万用表，型号为 179 , 测试结果见表 1 。

$\mathrm{MCP}$ 制作输入增强膜后, 面电阻比普通 MCP 略 有增加, 但面电阻在合格范围内 $(\leqslant 250 \Omega)^{[13]}$, 能 满足像增强器使用要求。

\section{表 $1 \mathrm{MCP}$ 输入面镀膜与不镀膜的面电阻比较}

Table 1 Comparison of the surface resistance of MCP input surface with and without input enhancement film

\begin{tabular}{ccc}
\hline MCP number & $\begin{array}{c}\text { MCP input } \\
\text { enhanced film }\end{array}$ & $\begin{array}{c}\text { Surface } \\
\text { resistance/ } \Omega\end{array}$ \\
\hline A-1 & with & 168 \\
A-2 & with & 147 \\
B-1 & without & 129 \\
B-2 & without & 110 \\
\hline
\end{tabular}

\section{2) XPS 能谱分析}

$\mathrm{MgO}$ 薄膜是碱性氧化物, 暴露在空气中容易吸收 水份和二氧化碳等物质。按照制管工艺流程, 输入增 强膜制作结束后不会马上进行使用, 需要在超净间内 流转约 4 24h。这就要求 $\mathrm{MgO}$ 薄膜吸收空气中的水 分、二氧化碳等物质后其成分不会发生变化, 所以采 用 XPS 对薄膜成分进行测试分析。

利用美国赛墨飞世尔科技公司 (Thermo fisher Scientific) 生产的型号为 K-Alpha ${ }^{+}$的 XPS 仪器。X 光
源为单色化的 $\mathrm{AlK} \alpha$ 源。分析室工作状态的真空度为 $2 \times 10^{-7} \mathrm{mbar}$, 束斑大小为 $400 \mu \mathrm{m}$, 扫描模式为固定 分析器能量 (CAE), 以表面吸附的 $\mathrm{C} 1 \mathrm{~s}$ (结合能: $284.8 \mathrm{eV}$ ) 为标准进行结合能校正, 图 2 是输入增强 膜的 XPS 能谱。

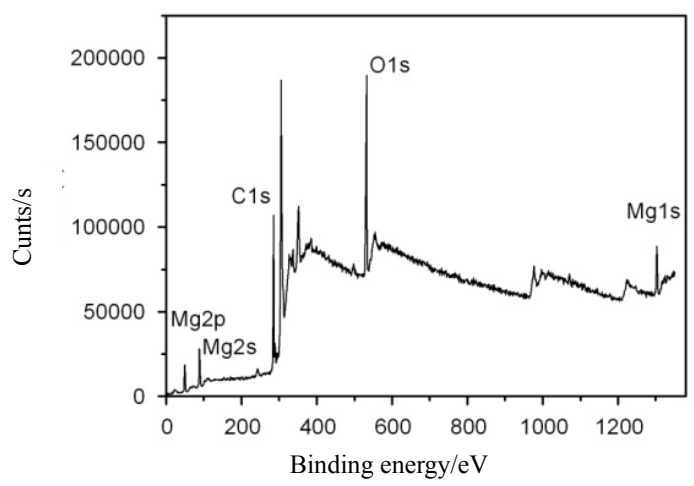

图 2 输入增强膜的 XPS 能谱

Fig.2 XPS spectrum of input enhanced film

由图 3 可以看出 $\mathrm{O} 1 \mathrm{~s}$ 和 $\mathrm{Mg} 2 \mathrm{p}$ 的峰值分别为 $531.20 \mathrm{eV}$ 和 $50.75 \mathrm{eV}$, 与 XPS 标准数据库 (National Institute of Standards and Technology, NIST) 中 $\mathrm{MgO}$ 的 $\mathrm{Mg}-\mathrm{O}$ 键结合能基本一致。通过灵敏度因子法可以 算出镁原子和氧原子的浓度比值 $(\mathrm{Mg}$ 的灵敏度因子 为 $0.12, \mathrm{O}$ 的灵敏度因子为 $0.66 ）$ 为 1.12 , 说明薄膜 在超净环境中存放 $24 \mathrm{~h}$, 其成分与 $\mathrm{MgO}$ 的标准成分 基本一致。

\section{3 输入增强膜对像增强器性能的影响}

\section{1 分辨力}

像增强器分辨力测试采用显微镜目视的方法。测 试系统由光源、中性滤光片、积分球、分辨力靶 (USAF1951)、准直镜、成像物镜、暗箱、显微镜等 组成 (如图 4 所示)。

USAF1951 分辨力靶是由美国空军于 1951 年创 建, 符合 MIL-STD-150A 的标准要求。分辨力靶是由 几组等宽的黑白相间的线条图案组成，各组线条的宽 度由宽逐渐变窄, 图案经成像物镜投射到像增强器的 光电阴极面上, 经像增强器倍增在苂光屏上显示出明 亮的分辨力靶板图像。由于像增强器的分辨力所限, 分辨力靶图像线条越窄越难分辨。把刚好能分辨出最 细线条细节的图案线对定义为分辨力, 单位为 $1 \mathrm{p} / \mathrm{mm}$ 。

像增强器的亮度增益调整为 $15000 \mathrm{~cd} \cdot \mathrm{m}^{-2} \cdot \mathrm{x}^{-1}$, 测试两组像增强器的分辨力, 结果见表 2 。 


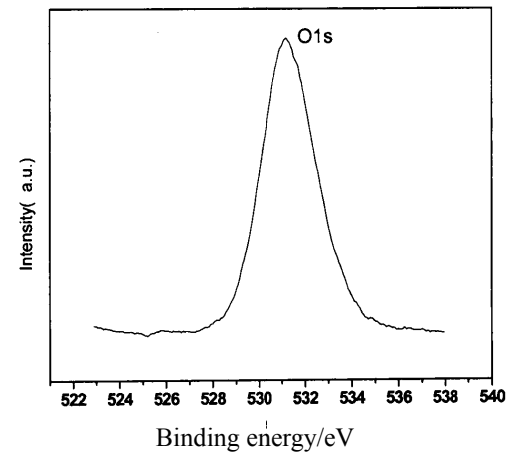

(a) O1s 谱峰 (a) O1s Spectral peak

图 $3 \mathrm{MgO}$ 输入增强膜 $\mathrm{O} 1 \mathrm{~s}$ 和 $\mathrm{Mg} 2 \mathrm{p}$ 谱峰

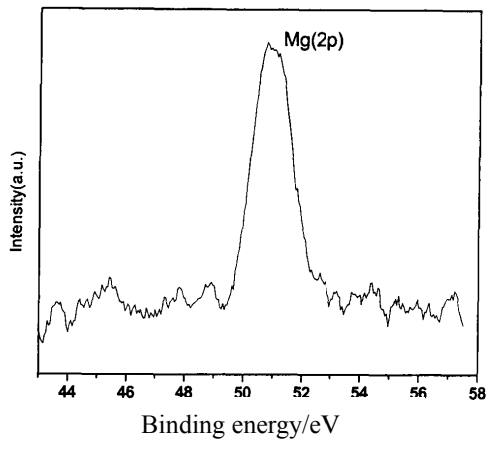

(b) Mg2p 谱峰 (b) Mg2p Spectral peak

Fig.3 O1s and $\mathrm{Mg} 2 \mathrm{p}$ peaks of $\mathrm{MgO}$ input enhanced films

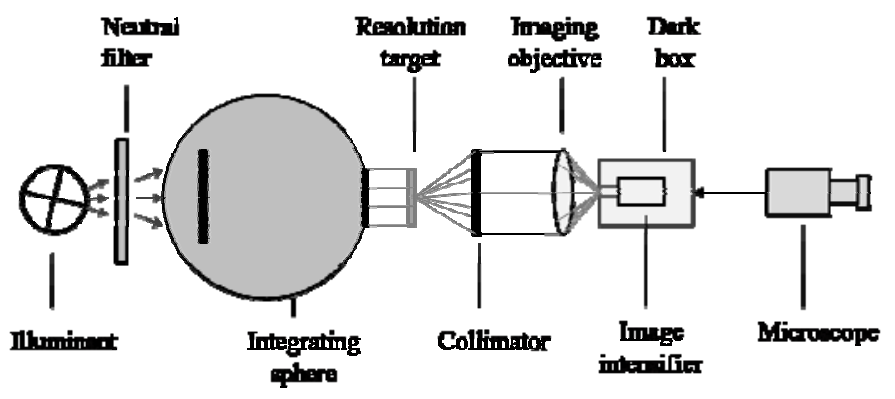

图 4 像增强器分辨力测试系统结构图

Fig.4 Diagram of resolution test set for image intensifier

表 2 两组像增强器的分辨力比较

Table 2 Contrast of resolution between two rinds of image intensifiers

\begin{tabular}{cccc}
\hline Number & $\begin{array}{c}\text { MCP } \\
\text { number }\end{array}$ & $\begin{array}{c}\text { MCP input } \\
\text { enhanced film }\end{array}$ & $\begin{array}{c}\text { Resolution } \\
/(\mathrm{lp} / \mathrm{mm})\end{array}$ \\
\hline 1\# & A-3 & with & 57 \\
$2 \#$ & A-4 & with & 54 \\
$3 \#$ & A-5 & with & 51 \\
$4 \#$ & A-6 & with & 57 \\
$5 \#$ & B-3 & without & 60 \\
$6 \#$ & B-4 & without & 64 \\
$7 \#$ & B-5 & without & 60 \\
$8 \#$ & B-6 & without & 60 \\
\hline
\end{tabular}

从表 2 可以看出, 无输入增膜的分辨力平均值为 $61 \mathrm{lp} / \mathrm{mm}$, 最大值为 $64 \mathrm{lp} / \mathrm{mm}$, 最小值为 $60 \mathrm{lp} / \mathrm{mm}$ 。 $\mathrm{MCP}$ 制作输入增强膜后, 分辨力平均值为 54.75 $\mathrm{lp} / \mathrm{mm}$, 最小值为 $51 \mathrm{lp} / \mathrm{mm}$, 最大值为 $57 \mathrm{lp} / \mathrm{mm}$ 。两 组像增强器的分辨力相比较, $\mathrm{A}$ 组像增强器的分辨力 下降约 $4 \mathrm{lp} / \mathrm{mm} \sim 10 \mathrm{lp} / \mathrm{mm}$ ，下降幅度达到 $10 \%$ 。

输入增强膜造成像增强器分辨力降低的原因是 $\mathrm{MCP}$ 输入端非开口区被激发出的二次电子数量增加, 使散射电子激增, 导致散射电子在荧光屏上生成的光 晕强度增强。2009 年顾燕针对散射电子对像增强器分
辨力影响展开了相应研究 ${ }^{[14]}$ 。研究结果表明 $\mathrm{MCP}$ 输 入端的散射电子会形成电子散射斑, 在像增强器的成 像图像上产生光晕效应, 导致分辨力下降影响像增强 器的成像质量。2016 年郭冰涛等人对到达 MCP 输入 面散射电子的偏移距离、运动轨迹进行了建模和仿真 计算 ${ }^{[15]}$ 。覆盖 $\mathrm{MCP}$ 输入端导电层制作输入增强膜后, 光电子激发输入增强膜产生的二次电子, 运动轨迹与 普通 $\mathrm{MCP}$ 输入端的散射电子相近。

$\mathrm{Ni}-\mathrm{Cr}$ 导电层的二次电子发射系数约为 0.97 , 而 $\mathrm{MgO}$ 输入增强膜的二次电子发射系数为 4.5 , 所以光 电子激发 $\mathrm{MgO}$ 输入增强膜发出的二次电子数量是光 电子激发 $\mathrm{Ni}-\mathrm{Cr}$ 导电层发出的二次电子数量的约 4.5 倍。MCP 制作输入增强膜后, 输入端的二次电子形成 在苂光屏上的发光强度大于 $\mathrm{Ni}-\mathrm{Cr}$ 导电层发出的二次 电子和弹性光电子形成的散射电子在苂光屏上的发 光强度, 所以 $\mathrm{MCP}$ 制作输入增强膜后像增强器的分 辨力会有明显的下降。

\subsection{MCP 增益}

因为电子的电荷量相同, 故在连续稳定工作状 态下 MCP 增益与电流增益相当, 所以可将 MCP 增 益定义为输出电子流与输入电子流之比 ${ }^{[16-17]}$, 计算 式如下:

$$
\mathrm{G}_{\mathrm{MCP}}=\frac{\left(I_{\mathrm{a}}-I_{\mathrm{a} 1}\right)}{\left(I_{\mathrm{c}}-I_{\mathrm{c} 1}\right)}
$$


式中: $G_{\mathrm{MCP}}$ 为 $\mathrm{MCP}$ 的电子流增益; $I_{\mathrm{a}}$ 和 $I_{\mathrm{a} 1}$ 分别表示 荧光屏电流 (输出电子流) 和荧光屏漏电流, $I_{\mathrm{c}}$ 和 $I_{\mathrm{c} 1}$ 分别表示光电阴极的电流（输入电子流）和漏电流。 $\mathrm{MCP}$ 增益测试原理如图 5 所示, 测试过程中, 光电阴 极的电压为 $-200 \mathrm{~V}, \mathrm{MCP}$ 电压为 $800 \mathrm{~V}$, 阳极电压 为 $6 \mathrm{kV}$ 。

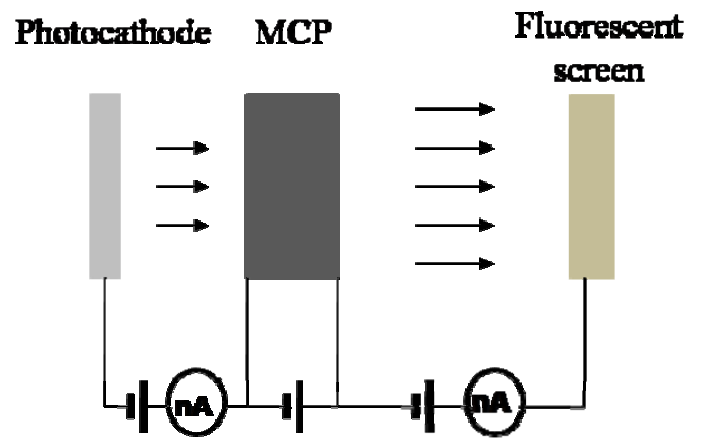

图 $5 \mathrm{MCP}$ 电流增益测试原理图

Fig.5 Diagram of MCP gain test set

按照该方法对 MCP 增益进行测试, 其结果见表 3。比较两组像增强器的 $\mathrm{MCP}$ 增益平均值, $\mathrm{A}$ 组像增 强器的 MCP 增益平均值是 450.5, B 组像增强器的 MCP 增益平均值是 209.5, MCP 镀膜后增益平均值比 没有镀膜的高 1.15 倍。实验用的 $\mathrm{MCP}$ 来源于同一屏 段, 每片 $\mathrm{MCP}$ 的增益基本相同, 所以 $\mathrm{A}$ 组像增强器 $\mathrm{MCP}$ 增益高出的部分主要是来自输入增强膜的贡献。

$\mathrm{A}$ 组和 $\mathrm{B}$ 组 $\mathrm{MCP}$ 的通道内壁的二次电子发射系 数相同, 所以 $\mathrm{MCP}$ 增益的高低直接受限于 $\mathrm{MCP}$ 对电 子的探测效率。2019 年杨露萍等人建立了 MCP 对电 子探测率的物理模型, 模拟分析了 $\mathrm{MCP}$ 开口区和非 开口区对电子探测率的贡献 ${ }^{[18]}$ 。借用他们的模型可以 计算出 MCP 非开口区制作输入增强膜后对电子的探 测率 $Q_{\mathrm{S}}$, 见式(2):

$$
Q_{\mathrm{S}}\left(E_{\mathrm{e}}, \theta\right)=\left(1-A_{\text {open }}\right) \cdot\left[1-\left(1-P_{1}\right)^{\delta_{0}\left(E_{\mathrm{e}}, \frac{\pi}{2}-\theta\right)}\right]
$$

式中: $P_{1}$ 为打到 $\mathrm{MCP}$ 输入面非开口区产生的二次电 子再次进入通道的平均几率 $\left(P_{1}\right.$ 的最大值与 $\mathrm{MCP}$ 的 开口面积比相同); $\delta_{0}$ 为输入面的二次电子发射系数; $A_{\text {open }}$ 为 $\mathrm{MCP}$ 的开口面积比 $(65 \%) ; E_{\mathrm{e}}$ 为电子能量, 此处为 $0.8 \mathrm{keV} ; \theta$ 为电子与 $\mathrm{MCP}$ 输入面法线的夹角。

$\mathrm{MCP}$ 制作输入增强膜后, $\delta_{0}$ 由 0.97 提高至 4.5 , 非开口区对电子的探测贡献也相应增大, 并且 $\mathrm{MCP}$ 通道壁前端的二次电子发射系数同时提高至 4.5 , 所 以输入增强膜可大幅提高 MCP 增益。

\section{3 信噪比}

在相同的条件下, 采用同一测试方法对像增强器 的信噪比展开测试, 测试结果见表 4 所示。从表 4 中 可以得出, A 组像增强器信噪比的平均值为 29.53 , B 组像增强器信噪比的平均值为 $25.27, \mathrm{MCP}$ 镀膜比不 镀膜的信噪比平均值高 4.26 , 增幅达到 $16.8 \%$ 。

当像增强器的灵敏度相同时 (如: $2 \#$ 和 $7 \# 、 4 \#$ 和 $8 \#$ ), 仍然是 MCP 镀膜的信噪比比 MCP 无膜的信 噪比高, 此时像增强器的信噪比提高主要是 MCP 噪 声因子 $F_{M}$ 减小了。从 $\mathrm{MCP}$ 噪声因子的计算表达式 (3) ${ }^{[19-20]}$ 可以看出, MCP 噪声因子主要受限于开口面 积比、首次碰撞的二次电子发射系数。 $\mathrm{MCP}$ 噪声因子 与像增强器信噪比的关系是 $\mathrm{MCP}$ 噪声因子越接近 1 像增强器的信噪比就越大, 反之则越小。

$$
F_{\mathrm{M}}=\frac{1}{R}\left[1+\frac{1}{\delta_{0}}+\left(\frac{1+\frac{G}{\delta_{0}}}{G}\right)\right]
$$

式中: $R$ 为 $\mathrm{MCP}$ 开口面积比; $\delta_{0}$ 为首次碰撞的二次 电子发射系数; $G$ 为 $\mathrm{MCP}$ 增益。

实验中 $\mathrm{A}$ 组和 $\mathrm{B}$ 组 $\mathrm{MCP}$ 的开口面积比相同，则

表 3 两组像增强器的 MCP 增益比较

Table 3 MCP gain comparison between two rinds of image intensifiers

\begin{tabular}{ccccc}
\hline Number & MCP number & MCP input enhanced film & Sensitivity $/(\mu \mathrm{A} / \mathrm{lm})$ & MCP gain \\
\hline $1 \#$ & A-3 & with & 888 & 421 \\
$2 \#$ & A-4 & with & 862 & 506 \\
$3 \#$ & A-5 & with & 806 & 389 \\
$4 \#$ & A-6 & with & 866 & 486 \\
$5 \#$ & B-3 & without & 796 & 213 \\
$6 \#$ & B-4 & without & 896 & 202 \\
$7 \#$ & B-5 & without & 862 & 200 \\
$8 \#$ & B-6 & without & 866 & 223 \\
\hline
\end{tabular}




\section{表 4 两组像增强器的信噪比比较}

Table 4 Comparison of signal to noise ratio between two rinds of image intensifiers

\begin{tabular}{ccccc}
\hline Number & MCP number & $\begin{array}{c}\text { MCP input } \\
\text { enhanced film }\end{array}$ & Sensitivity $(\mu \mathrm{A} / \mathrm{lm})$ & Signal to noise ratio \\
\hline 1\# & A-3 & with & 888 & 30.41 \\
$2 \#$ & A-4 & with & 862 & 29.15 \\
$3 \#$ & A-5 & with & 806 & 29.22 \\
$4 \#$ & A-6 & with & 866 & 29.35 \\
$5 \#$ & B-3 & without & 796 & 23.20 \\
$6 \#$ & B-4 & without & 896 & 25.59 \\
$7 \#$ & B-5 & without & 862 & 25.84 \\
$8 \#$ & B-6 & without & 866 & 25.43 \\
\hline
\end{tabular}

首次碰撞的二次电子发射系数越高、增益越大的 MCP 噪声因子就越小 (实验用 MCP 增益均远远大于 1 , 所 以噪声因子主要与输入面材料的二次电子发射系数 有关)。MCP 制作输入增强膜后, 输入面的二次电子 发射系数由 0.97 提高至 4.5, 带入(3)式计算, 该部分 的噪声因子由约 3.248 下降至 1.880。加之 $\mathrm{MCP}$ 非开 口区的导电层表面制作了输入增强膜, 部分光电子激 发出的二次电子也进入到了通道内, 同样提高了 $\mathrm{MCP}$ 的输入信号, 所以输入增强膜可以显著提高像增强器 的信噪比。

\section{4 结论}

为了研究提高 MCP 对电子的探测效率和倍增能 力, 采用真空镀膜的技术, 在 MCP 输入端导电层表 面镀制一定厚度、材料为 $\mathrm{MgO}$ 的输入增强膜。经过 实验对比, 输入增强膜可以提高像增强器的信噪比和 MCP 增益。但由于输入增强膜导致 MCP 输入端的散 射电子数量增加, 电子散射斑在荧光屏上的发光强度 增强, 导致分辨力降低。

因此, $\mathrm{MgO}$ 输入增强膜对于电子的倍增能力和探 测效率的提升具有良好的应用前景。如果将其应用于成 像观察中, 需考虑采取其他办法弥补所损失的分辨力。

\section{参考文献:}

[1] 向世明, 倪国强. 光电子成像器件原理[M]. 北京: 国防出版社, 2006. XIANG Shiming, NI Guoqiang. The Principle of Photoelectronic Imaging Devices[M]. Beijing: National Defense Press Industry Press, 2006.

[2] 白廷柱, 金伟其. 光电成像原理与技术[M]. 北京: 北京理工大学出版 社, 2006.

BAI Tingzhu, JIN Weiqi. Principle and Technology of Photoelectric Imaging[M]. Beijing: Press of Beijing University of Technology, 2006.
[3] 李晓峰. 三代像增强器[D]. 西安: 中国科学院西安光学精密机械研究 所, 2001.

LI Xiaofeng. Three Generation Image Intensifier[D]. Xi'an: Xi'an Institute of Optics and Precision Machinery, Chinese Academy of Sciences, 2001.

[4] 李晓峰, 张云昆, 许有毅, 等. 多碱光电阴极光电发射过程研究 [J]. 红 外技术, 2012, 34(8): 435-440.

LI Xiaofeng, ZHANG Yunkun, XU Youyi, et al. Study on photoemission process of multi-alkali cathode[J]. Infrared Technology, 2012, 34(8): $435-440$.

[5] 常本康. 多碱阴极光学监控原理研究 [J]. 真空科学与技术, 1994, 14(1): 41-46.

CHANG Benkang. A study of survey and control principles on optical information of multi-alkali photocathode[J]. Vacuum Science and Technology (CHINA), 1994, 14(1): 41-46.

[6] 赵恒, 常乐, 李廷涛, 等. 多碱光电阴极激活技术研究 $[\mathrm{J}]$. 红外技术, 2018, 40(7): 695-700.

ZHAO Heng, CHANG Le, LI Tingtao, et al. Study on Cs-O activation technology of multi-alkali photocathode[J]. Infrared Technology, 2018, 40(7): 695-700.

[7] 潘金生. 微通道板及其主要性能特征 [J]. 应用光学, 2004, 25(5): 25-29. PAN Jinsheng. Microchannel plates and its main characteristics[J]. Journal of Applied Optics, 2004, 25(5): 25-29.

[8] Joseph Ladislas Wiza. Microchannel plate detectors[J]. Nuclear Instruments and Methods, 1979, 162: 587-601.

[9] 郝子恒, 李相金釷, 张妮, 等. 微通道板高增益二次电子发射层制备研 究[J]. 红外技术, 2018, 40(11): 1077-1080.

HAO Ziheng, LI Xiangxin, ZHANG Ni, et al. Preparation of high gain secondary electron emission layer for microchannel plate[J]. Infrared Technology, 2018, 40(11): 1077-1080.

[10] Fraser G W. The electron detection efficiency of microchannel plates[J]. Nuclear Instruments and Methods, 1983, 206: 445-449.

[11] Koichi Kanaya. Secondary electron emission form insulators[J]. Appl. 
Phys., 1978(11): 2425-2437.

[12] 李晨, 罗宗泰, 王多书, 等. $\mathrm{MgO}$ 二次电子发射功能薄膜的制备方法 [J]. 真空与低温, 2009, 15(4): 187-192.

LI Cheng, LUO Zongsheng, WANG Duoshu, et al. Preparation methods of $\mathrm{MgO}$ thin films with secondary electron emission[J]. Vacuum and Cryogenics, 2009, 15(4): 187-192.

[13] 国防科学技术工业委员会. 微通道板规范: 第 3 部分要求 GJB 1596-93 [S]. 中华人民共和国国家军用标准, 1993.

National Defese Science Technology and Industry Commission. Specification for Microchannel Plate: Part Three Requirement GJB 1596-93[S]. Military Standards of the People's Republic of China, 1993.

[14] 顾燕. 电子散射对微光像增强器分辨力的影响研究[D]. 南京: 南京 理工大学, 2009.

GU Yan. Effect of Electron Scattering on Resolution of LLL Image Intensifier[D]. Nanjing: Nanjing University of Technology, 2009.

[15] 郭冰涛, 张卫国, 王键军, 等. 微光夜视系统光晕效应定量表征与建 模方法 [J]. 强激光与粒子束, 2016, 28(11): 164-169.

GUO Bingtao, ZHANG Weiguo, WANG Jianjun, et al. Modling and simulation of halo effect of night vision system[J]. High Power Laser and Particle Beams, 2016, 28(11): 164-169.

[16] 张小东, 欧阳晓平, 何军章, 等. 微通道板的增益研究[J]. 核技术, 2019, 10(42): 46-50.
ZHANG Xiaodong, OUYANG Xiaoping, HE Junzhang, et al. Gain study of microchannel plate[J]. Nuclear Techniques, 2019, 10(42): 46-50.

[17] 刘术林, 邓广绪, 严诚, 等. MCP 增益与首次电子能量关系的实验研 究[J]. 红外技术, 2011, 33(6): 354-365.

LIU Shulin, DENG Guangxu, YAN Cheng. Experiment research on research on relation between MCP gain and electron energy while first collision[J]. Infrared Technology, 2011, 33(6): 354-365.

[18] 杨露萍, 刘术林, 黄明举, 等. 提高微通道板对低能电子探测效率的 技术途径[J]. 红外技术, 2016, 38(8): 714-718.

YANG Luping, LIU Shulin, HUANG Mingju, et al. Technical approach to improve the detective efficiency of microchannel plate for low energy electron[J]. Infrared Technology, 2016, 38(8): 714-718.

[19] 向世明. 微光像增强器信噪比理论极限问题研究[J]. 应用光学, 2008, 29(5): 724-726

XIANG Shiming. Study on the theoretical limit of SNR of LLL image intensifier[J]. Journal of Applied Optics, 2008, 29(5): 724-726.

[20] 钱芸生, 常本康, 詹启海. 微光像增强器信噪比测试技术研究 [J]. 空科学与技术学报, 2002(5): 389-391.

QIAN Yunsheng, CHANG Benkang, ZHAN Qihai, et al. Development of signal-to-nose ratio tester for LLL image intensifier[J]. Vacuum Science and Technology, 2002(5): 389-391. 